\title{
CEREBELLAR PROJECTIONS TO THE SUPERIOR COLLICULUS IN THE CAT ${ }^{1}$
}

\author{
MANUELA ROLDÁN AND FERNANDO REINOSO-SUÁREZ ${ }^{2}$
}

Departamento de Morfología and Instituto de Investigaciones Oftalmológicas, Facultad de Medicina, Universidad Autónoma de Madrid, Madrid 34, Spain

\begin{abstract}
The projections from the cerebellar nuclei to the superior colliculus of the cat have been studied by injecting small amounts of horseradish peroxidase into the colliculus and charting the distribution of the labeled neurons in the cerebellar nuclei. The results show that the cerebellar nuclei project to the deep layers of the superior colliculus, especially contralaterally. Of the deep cerebellar nuclei, the lateral sends the most conspicuous projections to the superior colliculus. This projection is topographically organized in a rostrocaudal sense, ending principally in the intermediate layers, and is most dense in the rostral half of the contralateral superior colliculus. The lateral nucleus also projects to the rostral part of the ipsilateral superior colliculus. The nucleus interpositus anterior projects to the rostromedial part of the stratum griseum intermedium of the contralateral colliculus. The caudal pole of the fastigial nucleus projects to the strata grisea intermedium and profundum, mainly to the latter and specifically in the caudal half of both superior colliculi. These results demonstrate that, apart from the possible existence of direct cerebellar connections to the oculomotor nuclei, the visual cerebellum modulates, through monosynaptic projections, the activity of the deep layers of the superior colliculus which is related directly to the regulation of the eye- and headorienting movements.
\end{abstract}

Cerebellar afferents to the superior colliculus have been described in the cat using anterograde and retrograde techniques (Cohen et al., 1958; Voogd, 1964; Angaut and Bowsher, 1970; Lago, 1975; Edwards et al., 1979). However, there is no description in the literature of the place of origin, degree of participation, and site of termination in the colliculus of the fibers proceeding from each of the cerebellar nuclei. Moreover, the role played by the cat's superior colliculus in relation to eye and head orientation movements makes one suppose that there is an organized cerebellar projection to the colliculus.

This paper presents a study of the cerebellar projections to the superior colliculus using the horseradish peroxidase (HRP) neurohistochemical technique and demonstrating the existence of a topographical organization of these projections. It is part of a broader study of the afferent fiber connections of the superior colliculus, some of which have already been reported elsewhere ('Tortelly et al., 1980; Tortelly and Reinoso-Suárez, 1980).

\footnotetext{
${ }^{1}$ This work was supported by the Spanish "Comision Asesora de Investigación Científica y Técnica" Grant 3021/76. We wish to thank Josefina Hernández and Hortensia F. Lomana for help with the histological preparations and María Teresa F. Yuste for photographic assistance. We would also like to express our appreciation to Dr. Aloisio Tortelly for his help during the surgery.

${ }^{2}$ 'I'o whom correspondence should be addressed at Departamento de Morfología, Facultad de Medicina, Universidad Autónoma de Madrid, Arzobispo Morcillo, 2, Madrid 34, Spain.
}

\section{Materials and Methods}

Twenty adult cats, in which a 50\% HRP (Sigma type VI) aqueous solution was injected into the superior colliculus in different parts and at various depths, were used in the present study. The operations were performed under Nembutal anesthesia and the HRP solution was injected using a 1- $\mu$ l Hamilton syringe under direct visual control to avoid contamination of neighboring structures. For the localization of the injection sites, the ReinosoSuárez (1961) stereotaxic atlas of the cat brain was used. In one animal, six injections of $40 \mathrm{nl}$ were made superficially in different parts of the colliculus, leaving the peroxidase above the stratum opticum. In two animals, only one similar superficial injection of $40 \mathrm{nl}$ was made. In 16 cats, $40 \mathrm{nl}$ were injected in the stratum griseum intermedium and, in an additional cat, $80 \mathrm{nl}$ were injected at the same depth. After a survival time of 42 to $48 \mathrm{hr}$, the animals were perfused with a mixture of aldehydes under deep Nembutal anesthesia and the brains were processed according to the technique described by $\mathrm{Me}-$ sulam (1978) to demonstrate HRP-labeled neurons. The presence of HRP-positive cells in the cerebellar nuclei was indicated in drawings of sections of the cerebellum taken at equal intervals. Flood and Jansen's (1961) description was used for naming the cerebellar nuclei.

In all of the animals, the injected HRP remained confined to the colliculus with the exception of two cats with lateral and medial rostral injections, in which the 
injection halo spread to the caudal pole of the nucleus of the optic tract (lateral subdivision) and to the caudomedial pole of the nucleus pretectalis posterior, respectively.

\section{Results}

In the three animals with superficial injections, the enzyme remained above the stratum opticum and, in these cases, no labeled cells were observed in the cerebellar nuclei. In the remaining 17 animals, the injections were placed in the stratum griseum intermedium, extending down to the stratum griseum profundum in six of them. In all of these cases, labeled neurons appeared in the cerebellar nuclei, both ipsi- and contralaterally. The nuclei in which the neuronal labeling was most prominent, both in the number of positive cells and in the intensity of labeling, were the contralateral lateral cerebellar nucleus and, to a lesser extent, the nucleus fastigii. Ipsilaterally, only the nucleus fastigii exhibited labeled neurons in all cases, and sometimes, the number of ipsilateral cells was similar to the contralateral number. Also, the ipsilateral lateral cerebellar nucleus showed some labeled neurons; these were scarce in number and only appeared when the HRP was injected rostrally into the colliculus.

Most labeled neurons in the cerebellar nuclei were large and medium in size (22 to $34 \mu \mathrm{m}$ ), although some small labeled neurons (15 to $18 \mu \mathrm{m}$ ) were found also, mainly in the ventrolateral surface of the lateral nucleus. In all cases in which only one injection of $40 \mathrm{nl}$ was given, most neurons were labeled faintly; however, after injections were given rostrally in the colliculus, a significant number of neurons were labeled strongly.

The injections in the strata grisea intermedium and profundum of the colliculus were carried out in five different sites using at least two animals for each injection site (Figs. 1, 2, 4, and 5): (1) collicular central zone injections in an intermediate position, both in the anteroposterior and mediolateral dimensions; (2) rostral and lateral injections; (3) rostral and medial injections; (4) caudal and lateral injections; and (5) caudal and medial injections. Although the same amount of enzyme was injected in all cases, the topographical distribution and number of labeled neurons found in the cerebellar nuclei were different in each of these groups of animals. At first sight, these differences would allow the estimation of the relative degree to which each cerebellar nucleus projects to the superior colliculus, an issue on which we will comment further in the discussion. A caution against such an attempl arises, however, from the fact that the variations in retrograde labeling can result from different factors governing axonal uptake within the injected region (Nauta et al., 1974; Cowan and Cuenod, 1975; Cooke et al., 1975) which, unfortunately, are largely uncontrollable.

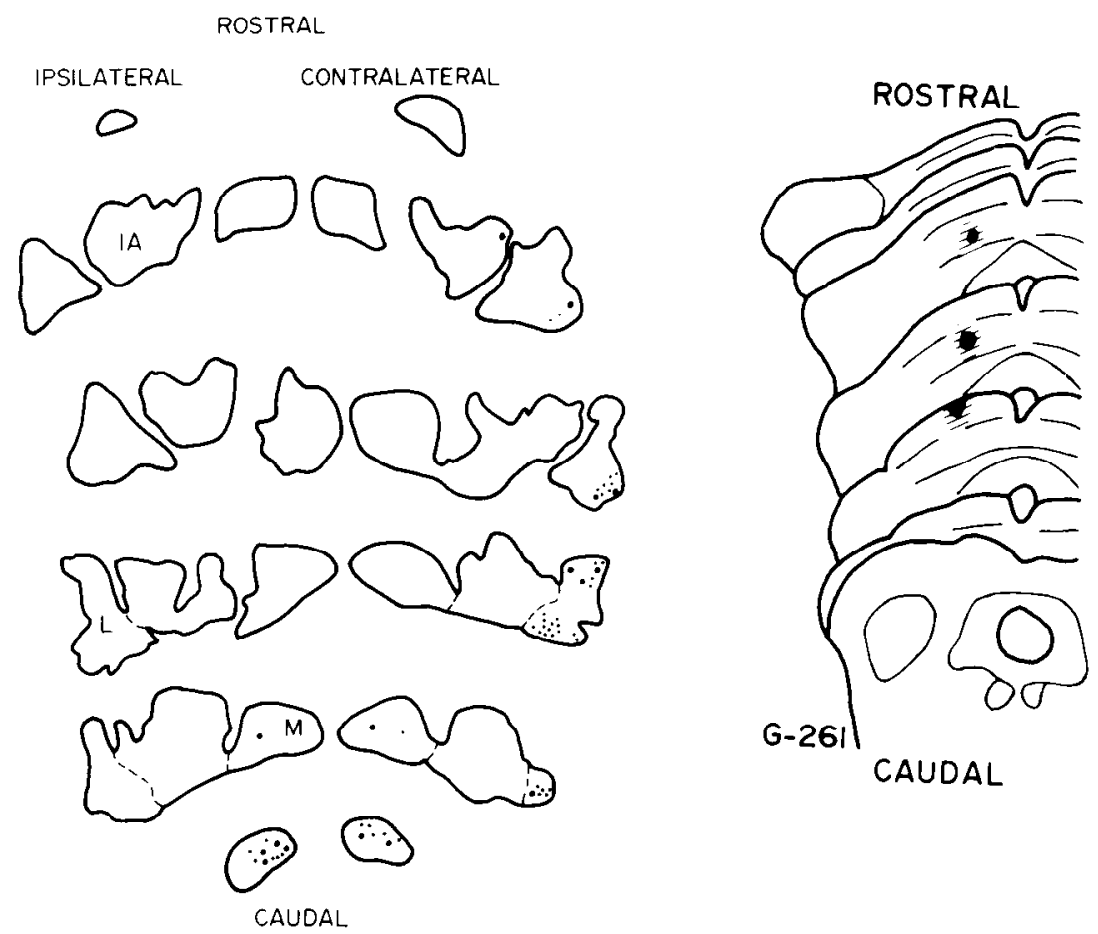

Figure 1. Case G-261. Diagrams showing the distribution of HRP-labeled neurons in the cerebellar nuclei and the injection site in the superior colliculus. The full extent of the HRP spread is plotted on a rostrocaudal series of coronal sections of the superior colliculus (right). In this case, the enzyme remained located in an intermediate position in the mediolateral dimensions of the colliculus, reaching in depth to the stratum griseum intermedium. In the drawing of the sections of the deep cerebellar nuclei (left), dots represent labeled cells in a one-to-one fashion. Individual neurons were grouped arbitrarily in two sets: those strongly labeled (large dots) and those faintly labeled (small dots). The abbreviations used are: $I A$, nucleus interpositus anterior; $L$, nucleus lateralis; $M$, nucleus fastigii. 
After the central injections (Fig. 1), only one-third of the positive cells appeared to be labeled strongly. The largest number of labeled cells was situated in the contralateral lateral nucleus, mostly ventrally, although we found some dorsally in the caudal part. Only in two animals was a neuron found in the anterior interpositus nucleus. Also, we found labeled neurons bilaterally in the caudal pole of the fastigius nucleus. The labeled neurons in the ipsilateral fastigius nucleus were situated preferentially in medial sectors of the nucleus (Figs. 1 and $5 F^{\prime}$ ).

The injections placed in the rostral part of the superior colliculus gave rise to the greatest number of labeled neurons in the cerebellar nuclei. The injections placed rostrally and laterally (Figs. $2 A$ and $3 A$ ) produced labeled neurons, the majority of which were labeled strongly in the ventrolateral part of the contralateral lateral nucleus (Fig. $5 B$ ). These neurons were found more rostrally than in the former group (central injections). Some neurons also could be found dorsally at caudal levels. A few labeled neurons could be found in the anterior and posterior interpositus nuclei. Some labeled neurons also were found in the ipsilateral lateral nucleus (Figs. $3 A$ and $5 E$ ). In the caudal pole of the medial nucleus, a few, slightly labeled neurons were found bilaterally, mostly in lateral sectors of the nucleus. These fastigial neurons were most abundant in those cases where the injection extended to the collicular stratum griseum profundum (Fig. $5 G$ ).

The injections situated medially and rostrally in the colliculus gave rise to a fair number of strongly labeled neurons, placed principally ventrally, laterally, and rostrally in the contralateral lateral nucleus (Figs. $2 B$ and $3 B$ ). A few HRP-positive neurons also were found in the dorsal and caudal part of the nucleus. The topography and intensity of the labeling in the contralateral lateral nucleus were very similar to the pattern found after rostral and lateral injections. Some labeled neurons were found laterally in the contralateral anterior interpositus nucleus and a smaller number were found in the rostral and lateral part of the posterior interpositus nucleus. Positive neurons found bilaterally in the medial nucleus were labeled only faintly and were in a medial position in the caudal pole of the nuclei (Fig. $3 B$ ).

The number of labeled neurons seen after injections placed caudally and laterally in the colliculus was small (Figs. $2 C$ and $3 C$ ). Most of them were located in the contralateral medial nucleus. We also found labeled neurons in the contralateral lateral nucleus, principally in its caudal half. Only a few neurons could be found in the caudal zone of the contralateral anterior interpositus nucleus and in the ipsilateral medial nucleus. Labeled neurons were found bilaterally in the caudal pole of the medial nuclei and these were predominantly in the lateral parts.

After HRP injections were placed medially and caudally in the superior colliculus (Figs. $2 D$ and $3 D$ ), labeled neurons were found caudally and ventrally in the contralateral lateral cerebellar nucleus (generally slightly labeled) and in the contralateral medial cerebellar nucleus. Some neurons were observed medially in the caudal pole of the ipsilateral medial nucleus. The strongly labeled neurons (most of which were situated in the fastigial nuclei) make up less than one-third of the positive HRP neurons in these cases. The pattern of distribution in the lateral nucleus is similar to that seen after the centrally placed injections but is limited to the caudal parts of the nucleus.

The above descriptions of the distribution of the labeled neurons in the cerebellar nuclei correspond to those of injections that were limited to the stratum griseum intermedium. Independent of the site of the colliculus in which they were placed, the injections that spread to the stratum griseum profundum gave rise to significantly more labeling, both in the number of cells and in the intensity of labeling, of HRP-positive neurons in the fastigial nuclei. Conversely, the neuronal labeling in the other cerebellar nuclei exhibited a pattern consonant with that described above for more superficially injected cases.

\section{Discussion}

These findings confirm the existence of projections to the superior colliculus from the cerebellar nuclei using silver impregnation methods and retrograde transport techniques in the cat as described previously (Cohen et al., 1958; Voogd, 1964; Angaut, 1970; Angaut and Bowsher, 1970; Lago, 1975; Edwards et al., 1979). Also, in accordance with these reports, they indicate that it is the strata grisea intermedium and profundum of the superior colliculus which receive the cerebellar projection since in none of the cases with injections superficial to the stratum opticum could labeled cells be found in the cerebellar nuclei.

Those injections that reached the stratum griseum profundum demonstrated neuronal labeling in greater numbers and intensity in the fastigial nuclei than injections in the same region which only reached the stratum griseum intermedium. This confirmed the descriptions of Angaut (1970) that, projections from the fastigial nucleus end in the stratum griseum profundum and are denser in the caudal part than in the rostral part since, in our material, the injections situated caudally in the colliculus also labeled neurons of the fastigial nuclei more intensely than did injections in the rostral part of the colliculus. The labeled neurons in the fastigial nucleus are more plentiful after medial than after lateral injections in the rostral part of the colliculus, confirming the findings of Angaut and Bowsher (1970) of denser terminal degeneration in the more medial region of the superior colliculus after lesioning the caudal pole of the fastigial nucleus. Likewise, after the medial injections, a greater number of labeled cells appeared in the ipsilateral medial nucleus, which agrees well with the above authors' descriptions of fibers ending at this level in the stratum griseum profundum after the fibers have recrossed the midline through the commissure of the superior colliculus. Although those authors described degenerating fibers only in the stratum griseum profundum, we have found labeled neurons in the caudal pole of the medial nucleus in animals in which injections were limited to the stratum griseum intermedium. Nonetheless, the labeling was certainly more conspicuous when the injections extended to the stratum griseum profundum. According to previous descriptions using other techniques, it is the posterior half of the medial cerebellar nucleus which projects to the superior colliculus (Cohen et al., 1958; Voogd, 1964; Angaut, 1970; 
A

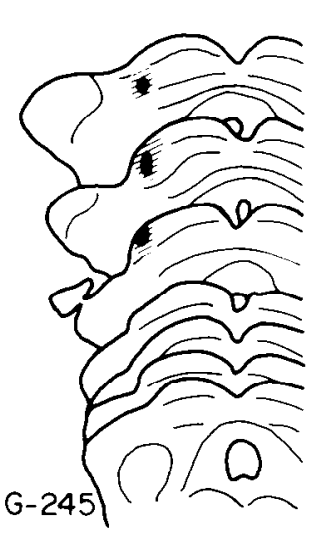

LATERAL
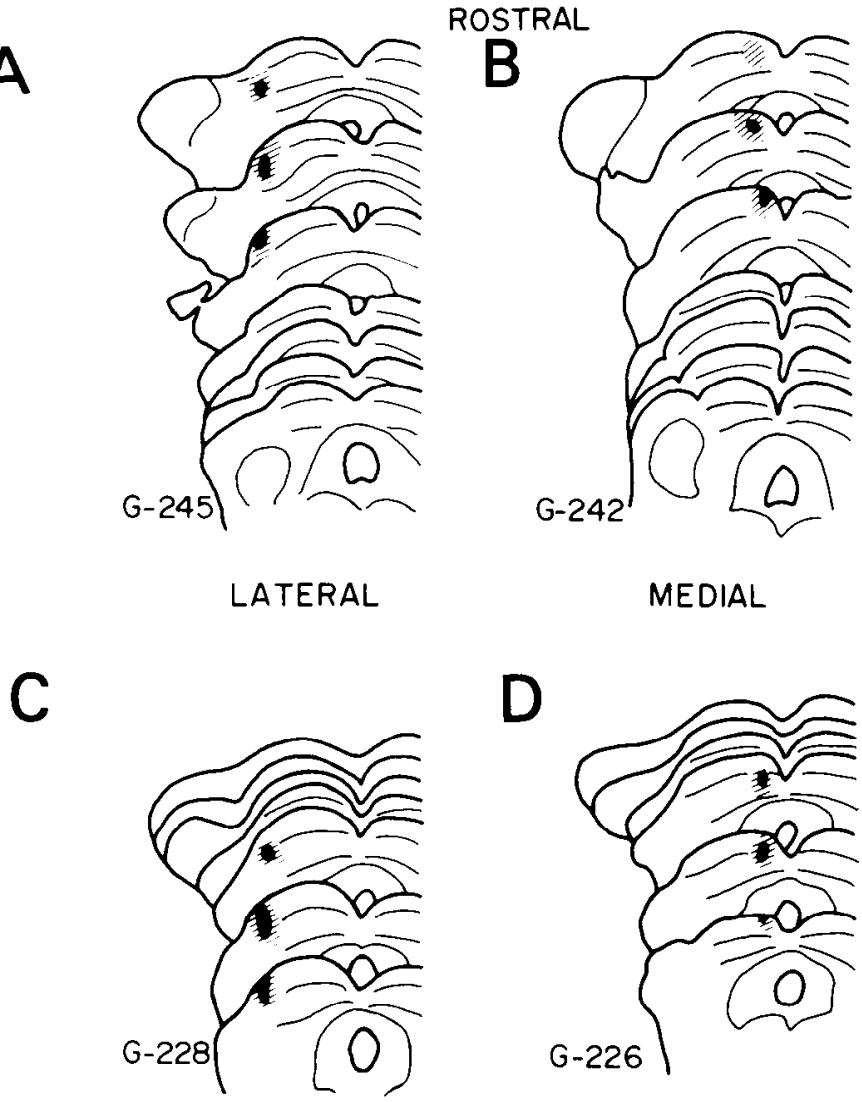

MEDIAL

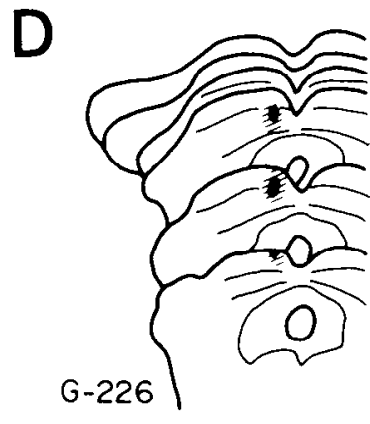

CAUDAL

Figure 2. Diagrams of coronal sections of the superior colliculus showing the rostrocaudal extent of the injection sites in the four animals whose labeled neurons are represented in Figure 3. See text for details.

Angaut and Bowsher, 1970). However, Edwards et al. (1979), using HRP, attribute these projections to the rostral part of this nucleus. In contrast to this, in all of our cases, the labeled neurons were found in the caudal pole of the nucleus; only a few scattered neurons were found at more rostral levels and these were always in the caudal third of the nucleus. Therefore, our findings do not confirm the claim of Angaut and Bowsher (1970) on the origin of the projection to the superior colliculus in the most rostral part of the caudal half of the nucleus. Furthermore, we show that there exists a certain topographical arrangement in the projections from the fastigius to the superior colliculus since the medial injections in colliculus tend to label neurons fundamentally in the medial part of the nucleus, and the intermediate or lateral injections produce labeled cells more laterally in the medial cerebellar nucleus.

The greatest number of labeled neurons in the interpositus nuclei was found in the anterior interpositus nucleus, and this was always after medial injections. The number of labeled cells and the intensity of labeling were the same after injections limited to stratum griseum intermedium and when the injections extended to the stratum griseum profundum. This shows that the projections of the anterior interpositus nucleus end predominantly in the medial and rostral parts of the stratum griseum intermedium of the contralateral colliculus. Only a few positive neurons were found in the laterorostral part of the posterior interpositus nucleus after injections in the rostral part of the colliculus, but these were never seen after caudal injections. These findings correlate with the negative results of Angaut (1970), who did not find projections from this nucleus to the colliculus using anterograde degeneration methods.

The lateral cerebellar nucleus sends the greatest number of projections to the superior colliculus. Most probably, these projections end in the stratum griseum intermedium of the anterior half since the injections situated superficial to this stratum do not label neurons in the cerebellar nuclei, and injections extending to the stratum griseum profundum do not increase the number of neurons labeled or the intensity of labeling in the lateral nucleus. These findings point to the lateral nucleus and, to a lesser extent, to the anterior interpositus as the origin of the degenerated fibers described by Lago (1975) as ending in the stratum griseum intermedium of the superior colliculus after lesions of the brachium conjunctivum in the cat. In particular, it is the ventral area of the whole lateral nucleus (ventrolateral in its anterior half) and, to a lesser extent, the dorsocaudal zone which project to the colliculus. These projections show an anterior to posterior topography, since the rostral injections label more neurons, and with greater intensity, more rostrally in the lateral nucleus. The intermediate injections label neurons faintly, with the majority situated in the intermediate parts of the contralateral lateral nucleus, and label fewer neurons than the rostral injections. The caudal injections in the colliculus label faintly an even smaller number of neurons and in a more caudal position in the lateral nucleus.

These findings demonstrate that not all of the cerebellar nuclei contribute in "almost" the same form to the cerebellocollicular projections, as was affirmed by Edwards et al. (1979), nor, at least in the superior colliculus, are the terminal fields of the fastigius, interpositus, and dentatus similar and overlapping as suggested by Cohen et al. (1958). We also demonstrate that it is the lateral nucleus (with a much less significant participation of the anterior interpositus) which is the non-medial nucleus projecting most widely to the colliculus-not the posterior interpositus nucleus as Voogd (1964) suggested. Finally, these findings demonstrate a topography in the origin of the projections in the cerebellar nuclei and in the termination in the superior colliculus.

We cannot rule out the possibility of spurious results caused by cells labeled by fibers passing through, but not terminating in, the $\mathrm{HRP}$ injection sites since there is evidence that the axons, injured by the injection procedure, take up and retrogradely transport HRP (Halperin and La Vail, 1975; Herkenham and Nauta, 1977). This would be quite important in the experiments in which the injections reach the stratum griseum profundum since, in this region, fibers have been described as proceeding from the cerebellar nuclei (Angaut, 1970) which penetrate laterally and pass to the opposite side, crossing the commissure of the superior colliculus (Angaut and Bowsher, 1970). However, the topography observed after our injections leads one to think that the labeled cells appearing in the cerebellar nuclei after collicular injec- 

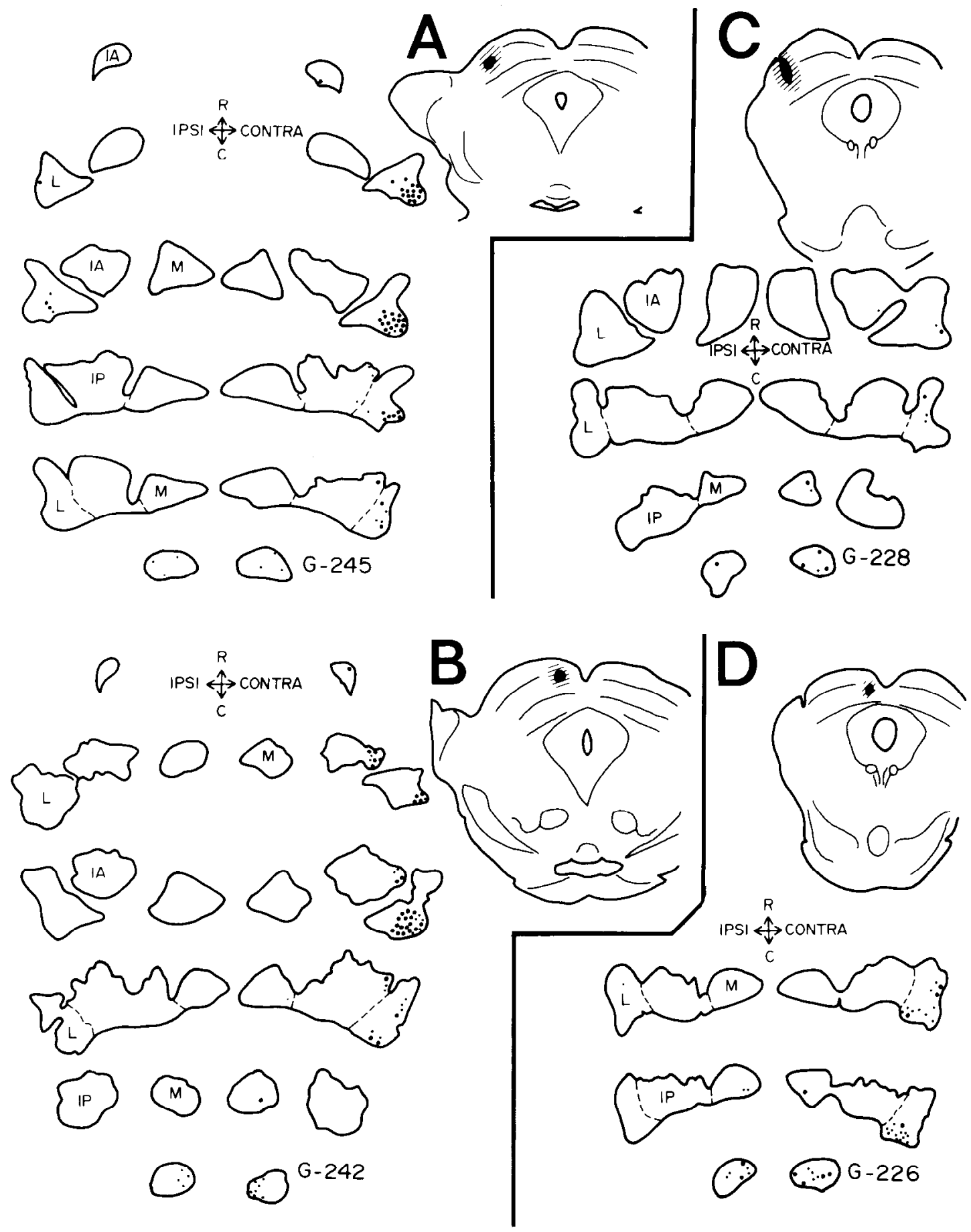

Figure 3. Diagrams, similar to those in Figure 1, showing the distribution of retrogradely labeled neurons in the cerebellar nuclei in the cases with injections in different locations in the superior colliculus that are represented in Figure 2. $A$, Rostrolateral injection (G-245); $B$, rostromedial injection $(\mathrm{G}-242) ; C$, caudolateral injection (G-228); $D$, caudomedial injection (G-226). The abbreviations used are: $C$, caudal; $C O N T R A$, contralateral side; $I A$, nucleus interpositus anterior; $I P$, nucleus interpositus posterior; $I P S I$, ipsilateral side; $L$, nucleus lateralis; $M$, nucleus fastigii; $R$, rostral.

tions must correspond largely to cerebellocollicular fibers which terminate in or near the locus of injection rather than to fibers of passage leading to other collicular territories. In the first place, the topography of the cerebellotectal projections shown here relies primarily on HRP injections which do not invade the deep layers of the colliculus wherein the crossing cerebellar fibers were described (Angaut and Bowsher, 1970). Furthermore, if such fibers were to actually cross through more superficial layers of the colliculus, the lateral injections should give a consistently larger number of positive cells in the contralateral cerebellar nuclei than the medial injections, which is clearly not the case.

Given the complicated role attributed to the superior 

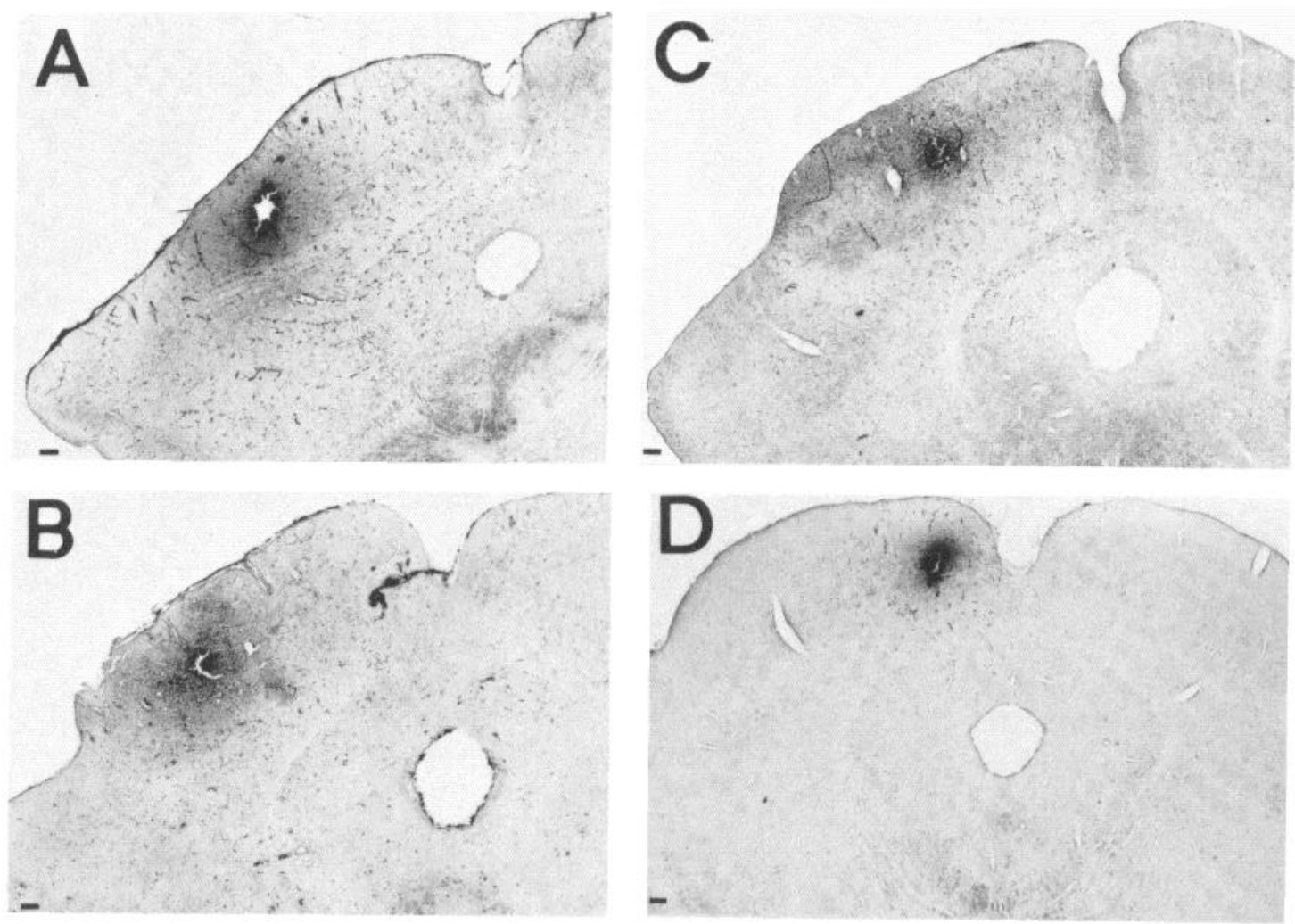

Figure 4. Low power photomicrographs of the superior colliculus showing the injection sites in four different animals. $A$ and $B$, Lateral injections; $C$, intermediate injection; $D$, medial injection.

colliculus in the organization of the movements of the eye and of other parts of the body (Sprague et al., 1973; Goldberg and Robinson, 1978), it is important that, within this organization, a cerebellar modulatory system is included since, as stated by Robinson (1976), the cerebellum is not so much an organ necessary for producing the movements as an organ necessary for making them correctly. The superior colliculi receive their projections from the caudal pole of the fastigial nuclei, in bilateral form, and the fibers are destined to the strata grisea intermedium and profundum, principally to the latter. This area of the fastigial nuclei receives, in turn, afferents from Larsell's VI and VII lobes, a vermal portion which is known to receive visual impulses and which is connected closely to the rhombencephalic structures participating in the regulation of eye movements (Snider and Stowell, 1942; Graybiel, 1974b; Llinás, 1974; Batini et al., 1978; Donaldson and Hawthorne, 1979). However, the principal connections of the superior colliculus come from the ventrolateral part of the lateral nucleus which projects essentially to the stratum griseum intermedium. It has been shown in the cat that this nucleus receives its afferent projections from the most lateral part of the anterior lobe, from the paramedial lobe, from crus I and crus II, and from the paraflocculus and flocculus (Voogd, 1964; Walberg and Jansen, 1964; Brodal and Courville, 1972; Courville et al., 1973; Dietrichs and Walberg, 1980). The area of the lateral nucleus which projects to the superior colliculus appears to correspond to the face region and, in a lesser proportion, to the hindlimb and forelimb regions (Voogd, 1964; Brodal and Courville,
1972; Courville et al., 1973). This ventrolateral region of the lateral nucleus receives projections principally from the paraflocculus and flocculus (Voogd, 1964; Angaut and Brodal, 1967; Yamamoto, 1978), cerebellar cortical formations that, in turn, receive direct afferents from the reticularis tegmenti pontis nucleus, pontine gray matter (Voogd, 1964; Hoddevik, 1978; Gerrits and Voogd, 1980), and inferior olive (Takeda and Maekawa, 1976). These brain stem structures are the link in the pathway mediating visual input from the tectum (Kawamura et al., 1974; Graham, 1977) and nuclei of the optic tract (Graybiel, 1974a; Takeda and Maekawa, 1976) to the ventrolateral area of the lateral cerebellar nuclei and these, in turn, as we demonstrate in this study, project upon the colliculus.

Cerebellar projections to the superior colliculus end in the deep layers, strata grisea intermedium and profundum of the colliculus. These are layers that project to the reticular formation, the oculomotor and/or perioculomotor nuclei, and the precerebellar formations (pontine reticular formation, pontine gray, and inferior olive). Further, they are the collicular structures responsible for activities such as the visual orientation responses (Casagrande et al., 1972). All of these facts provide stimulating insights into the complex cerebellar regulation of the organization of head and eye movements, a regulation which may be exerted over the nuclei directly implicated in the execution of movements (oculomotor complex) but which also must be made to act on structures, such as the superior colliculus, which exert a more sophisticated control over some special features of these movements. 

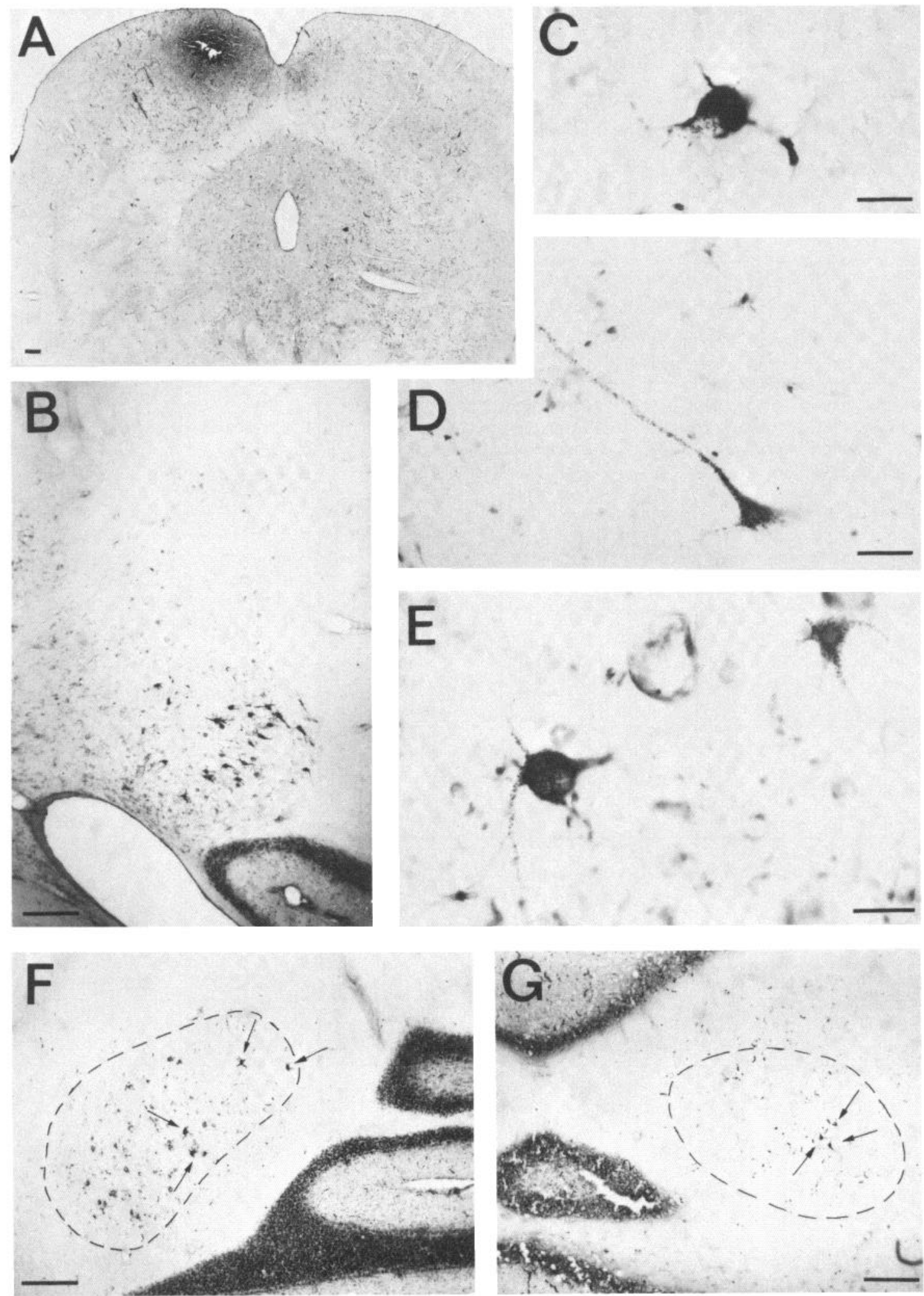

Figure 5. Photomicrographs showing the injection site and HRP-labeled neurons in various experimental cats. $A$, Injection site in a cat with a rostromedial injection. $B$ to $G$, Bright-field photomicrographs of HRP-labeled cells in the contralateral lateral cerebellar nucleus $(B)$, contralateral posterior interpositus nucleus $(C)$, contralateral anterior interpositus nucleus $(D)$, ipsilateral lateral cerebellar nucleus $(E)$, and the ipsilateral $(F)$ and contralateral $(G)$ caudal pole of the medial cerebellar nuclei. Scale line for $A, B, F$, and $G, 300 \mu \mathrm{m}$; for $C$ to $E, 30 \mu \mathrm{m}$. The arrows in $F$ and $G$ show strongly labeled neurons interspersed among faintly labeled neurons which are difficult to recognize at this magnification. 


\section{References}

Angaut, P. (1970) The ascending projections of the nucleus interpositus posterior of the cat cerebellum: An experimental anatomical study using silver impregnation methods. Brain Res. 24: 377-394.

Angaut, P., and D. Bowsher (1970) Ascending projections of the medial cerebellar (fastigial) nucleus: An experimental study in the cat. Brain Res. 24: 49-68.

Angaut, P., and A. Brodal (1967) The projection of the "vestibulocerebellum" onto the vestibular nuclei in the cat. Arch. Ital. Biol. 105: 441-479.

Batini, C., C. Buisseret-Delmas, J. Corvisier, O. Hardy, and D. Jassik-Gerschenfeld (1978) Brain stem nuclei giving fibres to lobules VI and VII of the cerebellar vermis. Brain Res. 153: 241-261.

Brodal, A., and J. Courville (1972) Cerebellar corticonuclear projection in the cat. Crus II. An experimental study with silver methods. Brain Res. 50: 1-23.

Casagrande, V. A., J. K. Harting, W. C. Hall, I. T. Diamond, and G. F. Martin (1972) Superior colliculus of the tree shrew: A structural and functional subdivision into superficial and deep layers. Science 177: 444-447.

Cohen, D., W. Chambers, and J. M. Sprague (1958) Experimental study of the efferent projections from the cerebellar nuclei to the brainstem of the cat. J. Comp. Neurol. 109: 233-259.

Cooke, C. T., P. U. Cameron, and D. G. Jones (1975) Stimulation-induced uptake of horseradish peroxidase by rat cortical synapses. Neurosci. Lett. 1: 15-18.

Courville, J., N. Diakiw, and A. Brodal (1973) Cerebellar corticonuclear projection in the cat. The paramedian lobule. An experimental study with silver methods. Brain Res. 50: 2545.

Cowan, W. M., and M. Cuenod (1975) The use of axonal transport for the study of neural connections: A retrospective survey. In The Use of Axonal Transport for Studies of Neuronal Connectivity, W. M. Cowan and M. Cuenod, eds., pp. 1-24, Elsevier, Amsterdam.

Dietrichs, E., and F. Walberg (1980) The cerebellar corticonuclear and nucleocortical projections of Crus I and Crus II. Neurosci. Lett. (Suppl.) 5: 439.

Donaldson, I. M. L., and M. E. Hawthorne (1979) Coding of visual information by units in the cat cerebellar vermis. Exp. Brain Res. 34: 27-48.

Edwards, S. B., G. L. Giusburgh, C. K. Henkel, and B. E. Stein (1979) Sources of subcortical projections to the superior colliculus in the cat. J. Comp. Neurol. 184: 309-330.

Flood, S., and J. Jansen (1961) On the cerebellar nuclei in the cat. Acta Anat. (Basel) 46:52-72.

Gerrits, N. M., and J. Voogd (1980) The pontine mossy fibre projections to the cerebellar flocculus in the cat. Neurosci. Lett. (Suppl.) 5: 440.

Goldberg, M. E., and D. L. Robinson (1978) Visual system: Superior colliculus. In Handbook of Behavioural Neurobiology, R. B. Masterton, ed., Vol. 1, pp. 119-164, Plenum Publishing Corp., New York.

Graham, J. (1977) An autoradiographic study of the efferent connections of the superior colliculus in the cat. J. Comp. Neurol. 173: 629-654.

Graybiel, A. M. (1974a) Some efferents of the pretectal region in the cat. Anat. Rec. 178: 365.

Graybiel, A. M. (1974b) Visuo-cerebellar and cerebello-visual connections involving the ventral lateral geniculate nucleus. Exp. Brain Res. 20: 303-306.
Halperin, J. J., and J. H. La Vail (1975) A study of the dynamics of retrograde transport and accumulation of horseradish peroxidase in injured neurons. Brain Res. 100: 253-269.

Herkenham, M., and W. J. H. Nauta (1977) Afferent connections of the habenular nuclei in the rat. A horseradish peroxidase study, with a note on the fibre-of-passage problem. J. Comp. Neurol. 173: 123-146.

Hoddevik, G. H. (1978) The projection from nucleus reticularis tegmenti pontis onto the cerebellum in the cat. A study using the methods of anterograde degeneration and retrograde axonal transport of horseradish peroxidase. Anat. Embryol. (Berl.) 153: 227-242.

Kawamura, K., A. Brodal, and G. Hoddevik (1974) The projection of the superior colliculus onto the reticular formation of the brain stem. An experimental anatomical study in the cat. Exp. Brain Res. 19: 1-19.

Lago, J. P. (1975) Proyecciones del cerebelo a través del pedúnculo cerebeloso superior. Trab. Inst. Cajal Invest. Biol. 67: 152.

Llinás, R. (1974) Motor aspects of cerebellar control. Physiologist 17: 19-46.

Mesulam, M. M. (1978) Tetramethyl benzidine for horseradish peroxidase neurohistochemistry: A non-carcinogenic blue reaction-product with superior sensitivity for visualizing neural afferent and efferents. J. Histochem. Cytochem. 26: 106-117.

Nauta, H. J. W., M. B. Pritz, and R. J. Lasek (1974) Afferents to the rat caudoputamen studied with horseradish peroxidase. An evaluation of a retrograde neuroanatomical research method. Brain Res. 67: 219-238.

Reinoso-Suárez, F. (1961) Topographischer Hirnatlas der Katze, E. Merck, A. G. Darmstadt, Germany.

Robinson, D. A. (1976) Adaptive gain control of the vestibular ocular reflex by the cerebellum. J. Neurophysiol. 39: 954-969.

Snider, R. S., and A. Stowell (1942) Evidence of a projection of the optic system to the cerebellum. Anat. Rec. 82: 448-449.

Sprague, J. M., G. Berlucchi, and G. Rizzolatti (1973) The role of the superior colliculus and pretectum in vision and visually guided behaviour. In Handbook of Sensory Physiology, $\mathrm{H}$. Antrum, R. Jung, W. R. Lowenstein, D. M. McKay, and H. L. Teuber, eds., pp. 27-101, Springer-Verlag, New York.

Takeda, T., and K. Maekawa (1976) 'The origin of the pretectoolivary tract. A study using the horseradish peroxidase method. Brain Res. 117: 319-325.

Tortelly, A., and F. Reinoso-Suárez (1980) Projections to the superior colliculus from the dorsal hypothalamic area and other prosencephalic structures derived from the embryonic subthalamic longitudinal band of the diencephalon. Neurosci. Lett. 18: 257-260.

Tortelly, A., F. Reinoso-Suárez, and A. Llamas (1980) Projections from non-visual cortical areas to the superior colliculus demonstrated by retrograde transport of HRP in the cat. Brain Res. 188: 543-549.

Voogd, J. (1964) The Cerebellum of the Cat. Structure and Fibre Connections, Van Gorcum \& Co., Assen, Netherlands.

Walberg, F., and J. Jansen (1964) Cerebellar corticonuclear projection studied experimentally with silver impregnation methods. J. Hirnforsch. 6: 338-354.

Yamamoto, M. (1978) Localisation of rabbit's flocculus Purkinje cells projecting to the cerebellar lateral nucleus and the nucleus prepositus hypoglossi investigated by means of the horseradish peroxidase retrograde axonal transport. Neurosci. Lett. 7: 197-202. 